\title{
Local experience with radiosurgery for vestibular schwannomas and recommendations for management
}

\author{
Ru Xin Wong ${ }^{1}$ MBBS, FRCR, Hui Ying Terese Low ${ }^{2}$, MBBS, MRCS, Daniel Yat Harn $\underline{\operatorname{Tan}}^{3}$, MBBS, FRCR
}

\begin{abstract}
INTRODUCTION There are many treatment options for vestibular schwannomas (VSs), including radiosurgery. Previous studies have shown good outcomes for smaller tumours. We report the results of a seven-year cohort of patients with VS who were treated at our centre using a linear accelerator-based stereotactic radiosurgery system.

METHODS We retrospectively reviewed the case notes and magnetic resonance (MR) images of patients with VS treated with radiosurgery. Treatment was administered as either a single 13 Gy session or 25 Gy in five sessions. At our centre, only larger or higher Koos grade VSs, were routinely treated with hypofractionated radiosurgery. Tumour response and hearing were assessed using RECIST criteria and Gardner-Robertson scale, respectively. Other toxicities were assessed using physical examination and history-taking. Freedom from radiological progression was estimated with the KaplanMeier method.

RESULTS 46 patients received single-fraction radiosurgery and 31 received hypofractionated radiosurgery. Median followup duration was 40.6 months. 29 patients had prior surgery to remove the tumour (median size $1.68 \mathrm{~cm}^{3}$ ). One patient who had symptomatic increase in tumour size ( $>20 \%$ in largest diameter) was treated conservatively and subsequently showed stable disease on MR imaging. Progression-free survival was $98.7 \%$. Another patient had symptomatic oedema requiring ventriculoperitoneal shunt insertion. 11 patients had serviceable hearing before radiotherapy and $72.7 \%$ of them retained useful hearing ( $20.1 \mathrm{~dB}$ mean decline in pure tone average). Facial and trigeminal nerve functions and sense of equilibrium were preserved in $>90 \%$ of patients.
\end{abstract}

CONCLUSION Radiosurgery is effective and safe for small VSs or as an adjunct therapy after microsurgery.

Keywords: acoustic neuroma, radiosurgery, radiotherapy, vestibular schwannoma

\section{INTRODUCTION}

Vestibular schwannomas (VSs) are benign tumours of the eighth cranial nerve. Treatment options include microsurgery, fractionated stereotactic radiotherapy (FSRT), stereotactic radiosurgery (RS), and a combination of surgery and radiotherapy (RT) or observation. Decision on treatment is made based on specific factors, such as the size of the VS, presenting symptoms, the patient's age, performance status and comorbidities, and whether the goal is hearing preservation. A recent Cochrane analysis, which aimed to look for randomised evidence in the treatment of VSs, concluded that therapy should be tailored to the individual, as the study could not find any high-quality evidence from randomised controlled trials. ${ }^{(1)}$ In a prospective study, Breivik et al showed that RS reduces tumour growth and extends treatment rate by tenfold, as compared with conservative management; they also found similar hearing preservation rates in both arms. ${ }^{(2)}$ To date, there are no randomised comparisons of RS versus surgery. Another systematic review, which analysed six controlled studies comparing RS and microsurgery, concluded that RS showed better outcomes for VS (up to $30 \mathrm{~mm}$ in cisternal diameter). ${ }^{(3)}$ Stereotactic RS comprises linear accelerator (LINAC)-based or gamma knife RS and FSRT. The Stereotactic Radiosurgery Task Force defines RS as high-dose hypofractionated RT performed for up to a maximum of five sessions. ${ }^{(4)}$ In theory, RS is more effective for local control, as it supposedly has a low $\alpha-\beta$ ratio for VS; however, it is also believed that fractionated RT has a better toxicity profile from a radiobiological viewpoint. No large randomised trial has addressed the differences in outcomes between RS and FSRT, although one large study of 449 patients, which pooled the results from three German centres, did show that both treatments were equally effective if the patients were chosen diligently based on tumour volume (the patients in the RS group had smaller tumours), pre-treatment characteristics and RS prescription $\leq 13 \mathrm{~Gy} .{ }^{(5)}$ To combine both the efficacy of singlefraction RS and the fractionated benefits of FSRT, hypofractionated treatment in 3-5 fractions has been proposed. This study was conducted to contribute our centre's experience with a LINACbased RS regimen (both single-session and hypofractionated).

\section{METHODS}

Between 2007 and 2014, 81 VS patients were treated with RS at the Department of Radiation Oncology, National Cancer Centre, Singapore. Only patients with follow-up data were included in the study. In total, we analysed 77 patients. The study was approved by the SingHealth Centralised Institutional Review Board.

In our institution, only smaller tumours or patients who are not keen on surgery are treated with RS. Patients with good, serviceable hearing are offered FSRT instead of RS in the belief that FSRT may be better for hearing preservation. Before treatment, all patients were seen by a multidisciplinary team

${ }^{1}$ Department of Radiation Oncology, National Cancer Centre Singapore, ${ }^{2}$ Department of Otorhinolaryngology, National University Hospital, ${ }^{3}$ Radiation Oncology, Asian American Medical Group, Gleneagles Hospital, Singapore

Correspondence: Dr Ru Xin Wong, Associate Consultant, Department of Radiation Oncology, National Cancer Centre Singapore, 11 Hospital Drive, Singapore 169610. Wong.ru.xin@singhealth.com.sg 
that consisted of neurosurgeons, ear, nose and throat surgeons, and radiation oncologists (with radiologist-reported magnetic resonance $[M R]$ imaging). Treatment decisions were made based on multidisciplinary consensus. Generally, patients were treated only if they were symptomatic (i.e. none of the 77 patients were 'incidentalomas'), and factors like comorbidities, life span and tumour growth rate were given consideration prior to treatment. Following resection, patients were treated only if the tumours showed progression. Standard T1 - and T2-weighted gadoliniumenhanced MR imaging with 1-mm slice thickness was carried out. For the purpose of immobilisation, a customised thermoplastic head frame was made for every patient.

The Brainlab ${ }^{\text {TM }}$ treatment planning system (Brainlab AG, Munich, Germany) was used and patients were treated with the Novalis ${ }^{\text {TM }}$ Shaped Beam Radiosurgery System (Brainlab AG), which was equipped with 3-mm micro-multileaf collimators and the ExacTrac image-guidance system (Brainlab AG). Finecut computed tomography simulation images were registered with recently acquired contrast-enhanced MR imaging and CISS (constructive interference in steady state) sequence MR images for target delineation. Both the radiation oncologists and neurosurgeons were involved in tumour contouring. A planning target volume (PTV) margin of $1-2 \mathrm{~mm}$ was given to account for intra-fraction motion and setup uncertainties. A marginal dose of either 12-13 Gy or 25 Gy in five fractions was prescribed to the PTV. An average of 7-9 conformal beams were used to create the treatment plan using a single isocentre. In cases where the lesion was abutting the brainstem or cochlear, intensity-modulated radiosurgery was used to achieve the dose constraints required. The majority of cases were normalised to the $80 \%$ isodose line, and the median conformality index in our cohort was 1.29 (maximum allowable <2). The minimum dose received by the PTV was $95 \%$ of the prescription dose, and no hotspots exceeding $100 \%$ of the prescription dose were allowed outside the PTV. Dose constraints were a maximum dose of 12 Gy to the brainstem and < 5 Gy to the ipsilateral cochlear if hearing preservation was a priority. For patients receiving $5 \times 5$ Gy fractions (hypofractionated RS), the brainstem constraint was $\mathrm{V} 23<0.5 \mathrm{~cm}^{3}$ and the maximum permissible dose limit was 30 Gy in five fractions. Image-guided RT tolerance was set to $0.7 \mathrm{~mm}$ and $1^{\circ}$ using the ExacTrac image-guidance system, and the patient was repositioned using the $6 \mathrm{D}$ robotic couch to correct for positioning errors, with final confirmatory ExacTrac verification before treatment was commenced. This process was repeated with every change in gantry angle to ensure accurate delivery. Fig. 1 shows dose colour-wash images of a treatment plan for a Koos Grade 4 VS, while Fig. 2 shows the corresponding dose-volume histogram for the brainstem. The maximum dose was $92.2 \%$, which is equivalent to $28.8 \mathrm{~Gy}$ and within the maximum permissible dose limit. Subjects were subsequently followed up with regular physical examination and routine MR imaging. Response Evaluation Criteria in Solid Tumors (RECIST) criteria $1.1^{(6)}$ was used for tumour response assessment, and progression was defined as an increase of $20 \%$ in the largest diameter of the lesion, including extension into the external auditory meatus.


Fig. 1 Dose colour-wash images show the treatment plan for a Koos Grade 4 tumour.

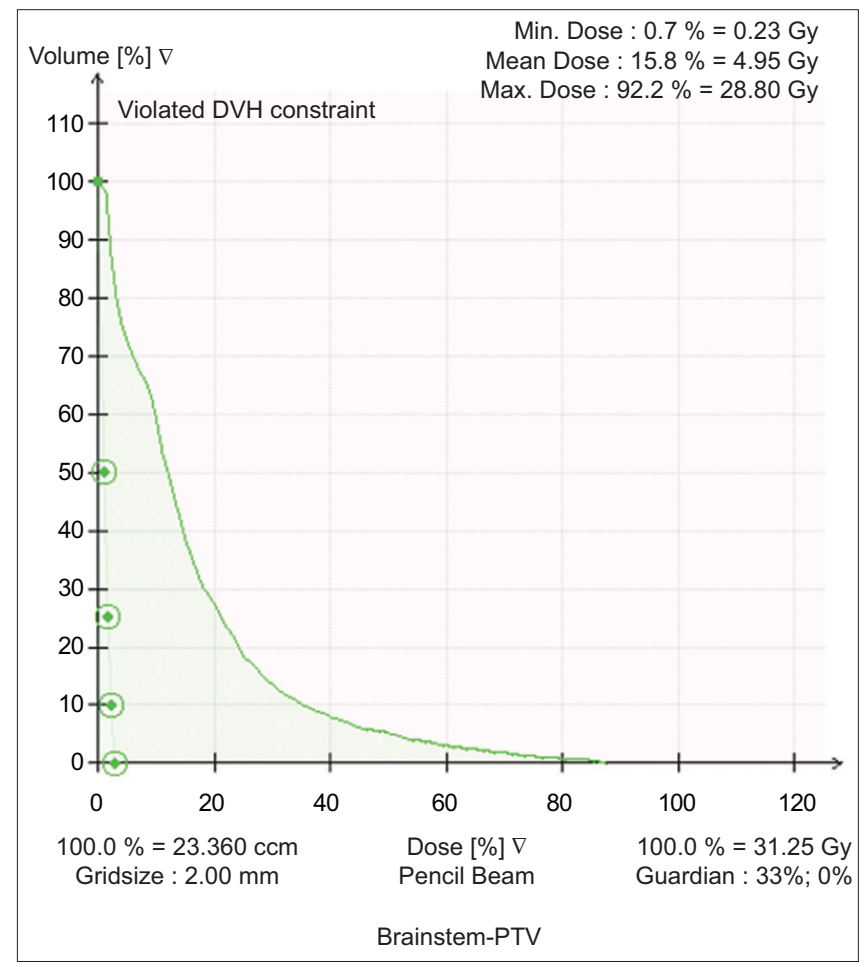

Fig. 2 Graph shows the dose-volume histogram for a Koos Stage 4 tumour treated with 25 Gy in five fractions. DVH: dose-volume histogram; PTV: planning target volume

This data was gathered from MR imaging reports and in some cases from review of the images. Post-treatment MR imaging was usually done in six-monthly intervals for the first $1-2$ years and yearly thereafter. The primary endpoint was freedom from progression, defined as time from end of RS to first progression based on RECIST criteria 1.1 on serial MR imaging intervention. Secondary endpoints included freedom from: (a) surgery (defined as time from end of RS to first surgical intervention, including shunt placement or resection of tumour); (b) hearing, trigeminal 
and facial nerve dysfunctions; and (c) symptoms of disequilibrium. Hearing was reported using the Gardner-Robertson scale with both clinical history and audiograms if available. Serviceable hearing was defined as the ability to use the telephone with the affected ear, or a pure-tone average (PTA) $\leq 50 \mathrm{~dB}$. Other toxicities, such as facial numbness and disequilibrium, were recorded with the patient's subjective reporting. Assessment was usually performed within a few weeks of commencement of RS.

Statistical analysis was performed with IBM SPSS Statistics version 19.0 (IBM Corp, Armonk, NY, USA). Freedom from progression and surgery outcomes were assessed using the Kaplan-Meier method.

\section{RESULTS}

A total of 77 patients were treated with RS. Table I shows their pretreatment characteristics. Of these 77 , three had neurofibromatosis Type 2 (NF-2). 46 patients received singlefraction treatment and 31 received hypofractionated treatment. The mean age of the patients was 53.7 (range 17-78) years. Median follow-up duration was 40.6 (range 4.6-90.7, interquartile range 40.3 ) months. Median tumour size was $1.68 \pm 3.75$ (range $0.14-23.52$ ) $\mathrm{cm}^{3}$. Median tumour size for single-fraction and hypofractionated regimes were $1.18 \mathrm{~cm}^{3}$ and $3.12 \mathrm{~cm}^{3}$, respectively. One patient whose tumour was $23.52 \mathrm{~cm}^{3}$ received hypofractionated treatment due to poor performance status. He was not eligible for resection and found it logistically difficult to attend multiple sessions of FSRT; pre-treatment, he had received a ventriculoperitoneal (VP) shunt insertion.

In all, 36 patients were in Koos Grade 3 or 4 (i.e. the tumour was in contact with or compressing the brainstem). Out of the 46 patients who received single-fraction treatment, 16 (34.8\%) were Koos Grade 3 or 4 . Among the 31 who received hypofractionated treatment, $20(64.5 \%)$ were Koos Grade 3 or 4. 29 patients had prior surgery -23 received radiation after subtotal resection, and six had tumour progression despite gross total resection. 48 patients did not have prior resection of tumour 29 received RT due to progression despite an initial period of observation and 19 received RT upon diagnosis. None of the 77 cases were 'incidentalomas' who had exhibited symptoms that prompted further investigations and subsequent treatment. Only 11 of the 77 patients had pre-RT serviceable hearing (i.e. PTA $\leq 50 \mathrm{~dB}$ or able to use the telephone with the affected ear). Prior to RS, 20 patients had facial nerve palsy, 18 had trigeminal nerve palsy and 50 had disequilibrium. Of the 20 with facial nerve palsy, all had undergone prior resection.

Following RS, none of the 46 patients who received singlefraction treatment had progression of tumour or required surgery. Of the 31 who received hypofractionated treatment, one had radiological progression (Table II) and one required a VP shunt insertion for peritumoral oedema. The patient who had progression of tumour on MR imaging also experienced worsening facial nerve palsy 11 months after RS. Although her initial average tumour size was $1.55 \mathrm{~cm}^{3}$, it was abutting the right middle cerebellar peduncle. Post-RS MR imaging showed that the tumour had become more heterogeneous, in keeping with
Table I. Pre-treatment characteristics of patients $(n=77)$.

\begin{tabular}{|c|c|}
\hline Variable & No. (\%) \\
\hline Age $^{*}(y r)$ & $53.7(17-78)$ \\
\hline Follow-up duration* (mth) & $40.6(4.6-90.7)$ \\
\hline \multicolumn{2}{|l|}{ Type of radiosurgery } \\
\hline Single-fraction & $46(59.7)$ \\
\hline $25 \mathrm{~Gy} / 5$ fractions & $31(40.3)$ \\
\hline Tumour size ${ }^{\dagger}\left(\mathrm{cm}^{3}\right)$ & $1.68(0.14-23.52)$ \\
\hline Single-fraction & 1.18 \\
\hline Hypofractionated & 3.12 \\
\hline \multicolumn{2}{|l|}{ Koos grade } \\
\hline \multicolumn{2}{|l|}{ Single-fraction } \\
\hline 1 or 2 & $30(65.2)$ \\
\hline 3 or 4 & $16(34.8)$ \\
\hline \multicolumn{2}{|l|}{ Hypofractionated } \\
\hline 1 or 2 & $11(35.5)$ \\
\hline 3 or 4 & $20(64.5)$ \\
\hline \multicolumn{2}{|l|}{ Previous excision } \\
\hline Yes & $29(37.7)$ \\
\hline No & $48(62.3)$ \\
\hline \multicolumn{2}{|l|}{ Indication for radiotherapy } \\
\hline Adjuvant after subtotal resection & $23(29.9)$ \\
\hline $\begin{array}{l}\text { Progression after gross total } \\
\text { resection }\end{array}$ & $6(7.8)$ \\
\hline Progression after initial observation & $29(37.7)$ \\
\hline Upon diagnosis & $19(24.7)$ \\
\hline \multicolumn{2}{|l|}{ Hearing } \\
\hline Serviceable & $11(14.3)$ \\
\hline Non-serviceable & $66(85.7)$ \\
\hline \multicolumn{2}{|l|}{ Facial nerve palsy } \\
\hline Yes & $20(26.0)$ \\
\hline No & $57(74.0)$ \\
\hline \multicolumn{2}{|l|}{ Trigeminal nerve palsy } \\
\hline Yes & $18(23.4)$ \\
\hline No & $59(76.6)$ \\
\hline \multicolumn{2}{|l|}{ Disequilibrium } \\
\hline Yes & $50(35.1)$ \\
\hline No & 27 (64.9) \\
\hline
\end{tabular}

Data presented as *mean (range) and tmedian (range).

Table II. Post-radiosurgery response on magnetic resonance (MR) imaging $(\mathbf{n}=\mathbf{7 7})$.

\begin{tabular}{|ll|}
\hline MR imaging response & No. (\%) \\
\hline Progression & $1(1.3)$ \\
\hline Partial response & $28(36.4)$ \\
\hline Stable disease & $48(62.3)$ \\
\hline
\end{tabular}

treatment response, although the absolute dimensions were larger. The patient was managed conservatively and subsequent followup imaging showed stable disease. Freedom from radiological progression was seen in $98.7 \%$ of patients $(95 \%$ confidence interval [Cl] 95.8\%-100\%; Fig. 3). The patient who had developed symptomatic peritumoral oedema underwent VP shunt insertion 


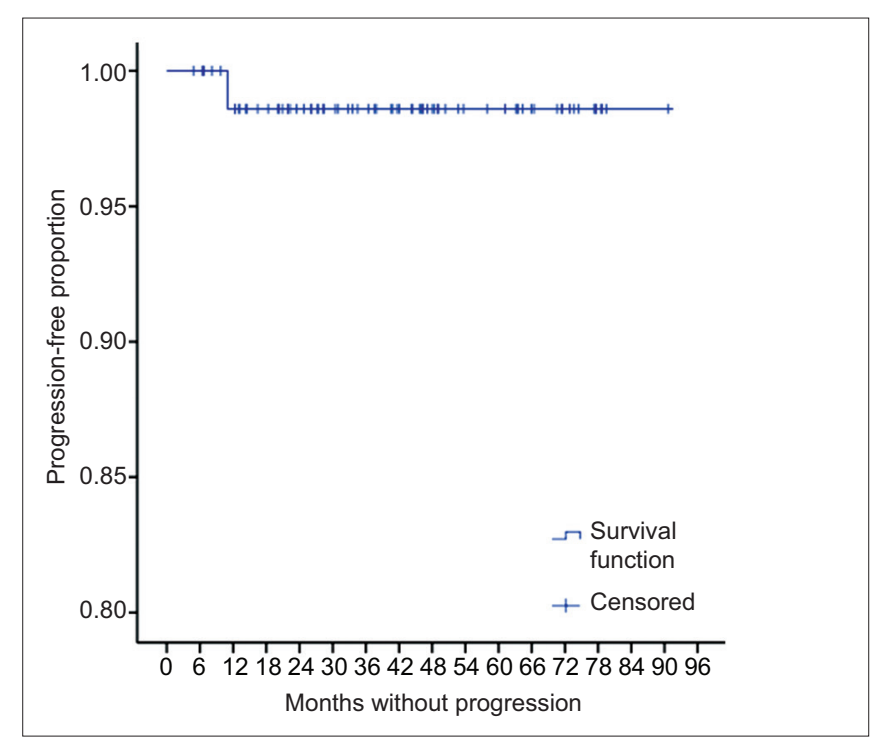

Fig. 3 Graph shows the proportion of progression-free patients.

for hydrocephalus at 15 months after hypofractionated treatment. This patient had a larger-than-median tumour size of $7.17 \mathrm{~cm}^{3}$, and the tumour was initially indenting the pons. Freedom from surgery was seen in $98.7 \%$ of patients (95\% Cl 95.2\%-100\%).

The majority of patients retained good cranial nerve function after RS (Table III). Among the 11 patients with pre-RT serviceable hearing, $8(72.7 \%)$ had retained serviceable hearing. Hearing preservation rate was $100 \%(n=5)$ in the single-fraction treatment group and $50 \%(\mathrm{n}=3)$ in the hypofractionated treatment group. Among those who had both available pre- and post-RT audiogram results, the mean decrease in PTA was $20.1 \mathrm{~dB}$. Facial and trigeminal nerve functions and sense of equilibrium were preserved in more than $90 \%$ of patients. No patient developed secondary neoplasms over the course of follow-up.

\section{DISCUSSION}

In our institution, only patients with smaller tumours are offered RS. Larger tumours receive either FSRT, or surgery upfront with or without adjuvant RS. For Koos Grade 3 or 4 tumours, we tend to offer hypofractionated instead of single-fraction RS. This study has shown that with careful patient selection - especially with regard to tumour size - patient outcomes were excellent, with freedom from progression and freedom from surgery both at $98.7 \%$. Many other larger studies have also confirmed the safety of single-fraction RS, ${ }^{(5,7-10)}$ although far fewer and smaller studies have reported on hypofractionated RS..$^{(11-14)}$

Our patient who had peritumoral oedema requiring VP shunt insertion had a larger tumour $\left(7.17 \mathrm{~cm}^{3}\right.$, median $\left.1.68 \mathrm{~cm}^{3}\right)$ prior to RS, and this could have been a predisposing factor for complications. Chan et al's study ${ }^{(9)}$ on FSRT found that tumour size was strongly predictive of neurosurgical complications, and that three- and five-year actuarial rates of freedom from any neurosurgical intervention were $100 \%$ and $97 \%$ for patients with tumour volume $<8 \mathrm{~cm}^{3}$, but were $74 \%$ and $47 \%$ for tumour volume $>8 \mathrm{~cm}^{3}$. The authors also recommended that large tumours be debulked maximally while preserving cranial nerves and that adjuvant RS should be given for subtotal resection or for
Table III. Post-radiosurgery cranial nerve function of patients who had single-fraction vs. hypofractionated radiosurgery.

\begin{tabular}{|c|c|c|}
\hline \multirow[t]{2}{*}{ Variable } & \multicolumn{2}{|c|}{ No. (\%) } \\
\hline & $\begin{array}{l}\text { Single-fraction } \\
(n=46)\end{array}$ & $\begin{array}{l}\text { Hypofractionated } \\
(n=31)\end{array}$ \\
\hline \multicolumn{3}{|l|}{ Hearing* } \\
\hline Serviceable & $5(100)$ & $3(50)$ \\
\hline Non-serviceable & 0 & $3(50)$ \\
\hline \multicolumn{3}{|c|}{$\begin{array}{l}\text { New/worsening facial } \\
\text { nerve dysfunction }\end{array}$} \\
\hline Yes & $1(2.2)$ & $2(6.5)$ \\
\hline No & 45 (97.8) & $29(93.5)$ \\
\hline \multicolumn{3}{|l|}{$\begin{array}{l}\text { New/worsening } \\
\text { trigeminal nerve } \\
\text { dysfunction }\end{array}$} \\
\hline Yes & $1(2.2)$ & $2(6.5)$ \\
\hline No & $45(97.8)$ & $29(93.5)$ \\
\hline \multicolumn{3}{|l|}{$\begin{array}{l}\text { Worsening } \\
\text { disequilibrium }\end{array}$} \\
\hline Yes & $1(2.2)$ & $1(3.2)$ \\
\hline No & 45 (97.8) & $30(96.8)$ \\
\hline
\end{tabular}

*Among those with serviceable hearing before radiosurgery ( $n=11$, 5 single-fraction and 6 hypofractionated).

tumour regrowth following gross total resection. ${ }^{(9)}$ This approach has been supported by Iwai et al, ${ }^{(15)}$ who published a paper on a series of 40 patients with large tumours (median maximum diameter $32.5 \mathrm{~mm}$ ). In this study, the authors reported that planned partial surgical resections followed by gamma knife RS had a good tumour control rate of $86 \%$ and a high rate of facial nerve and hearing preservation. However, in another study comprising a cyberknife series of 33 VSs with tumour volume $>8 \mathrm{~cm}^{3}$ treated with 2-5 fractions (14.0-19.5 Gy), only two patients were reported to require debulking, with another two requiring shunts. ${ }^{(16)}$ Another study by Aoyama et $\mathrm{al}^{(17)}$ showed that size was predictive of tumour progression. Their series, which included 201 patients who were treated with FSRT, reported tumour expansion rates of $0 \%, 11.4 \%, 25.6 \%$ and $50 \%$ in tumours measuring $9 \mathrm{~mm}, 10-19 \mathrm{~mm}, 20-29 \mathrm{~mm}$ and $30 \mathrm{~mm}$, respectively; in all, only $5 \%$ of patients required surgical salvage. ${ }^{(17)}$ In our study, although the patient with radiological progression had a small tumour $\left(1.55 \mathrm{~cm}^{3}\right)$, there was a transient increase in size with resultant facial nerve compression, probably due to treatment reaction. This patient was managed conservatively without further symptoms or tumour progression. The phenomenon of transient increase in tumour size has been reported previously; a Swedish study from Karolinska Institutet, comprising a series of 254 patients, reported that $12 \%$ of VSs continued to enlarge temporarily over 6-18 months after treatment and subsequently either shrank or stabilised. ${ }^{(18)}$ However, it is not clear what the definition of size increase was in this Swedish study. Another large series of 496 patients from John Hopkins Hospital, ${ }^{(19)}$ in which the majority were treated with 25 Gy over five fractions, reported that $30 \%$ of patients experienced radiologic progression. In the John Hopkins study, radiologic progression was defined as 
"tumour volume greater than the baseline volume and a stable clinical status at follow-up ${ }^{\prime(19)}$ and the method for assessing tumour volume also differed from that used in the current study. In our study, only the presence of tumours that fulfilled RECIST criteria 1.1 for progression were defined as progression. All in all, we recommend that early mild progression be monitored with serial MR imaging instead of immediate resection.

In our series, 36 out of 77 patients had Koos Grade 3 or 4 tumours. Koos Grade 4 tumour is defined as a tumour with extension to the external auditory canal $>30 \mathrm{~mm}$ or causing brainstem compression. This definition is not absolutely clear, as brainstem compression runs a spectrum from mild indentation of the brainstem to gross contortion of the brainstem and fourth ventricle. ${ }^{(20)}$ Although the clinical outcome was excellent in our current study, it has also been reported that brainstem compression is a risk factor for progression. Hasegawa et al's study of 440 gamma knife surgical patients found that brainstem compression with fourth ventricle compression significantly resulted in worse outcome. ${ }^{(10)}$ Another smaller report of 65 patients with larger-volume VS by Yang et $\mathrm{al}^{(21)}$ similarly found that Koos Grade 4 tumours were less likely to have tumour control; in that study, nine out of the 65 patients had larger tumour volumes requiring intervention. Their median tumour volume of $9 \mathrm{~cm}^{3}$, which is much larger than that of our series, could explain the higher rate of complications. ${ }^{(21)}$ In our institution, gross brainstem or fourth ventricular distortion with symptomatic mass effect is a relative contraindication for $\mathrm{RS}$.

Only 11 patients in our study had pre-RS serviceable hearing. This is because our institution tends to offer FSRT to patients who have good hearing prior to RT. Among these 11 patients, the rate of hearing preservation was $72.7 \%$ and the decline in mean PTA was $20.1 \mathrm{~dB}$. A Japanese series also reported a similar mean PTA decline of $27.3 \mathrm{~dB} \cdot{ }^{(14)}$ An analysis of 4,234 patients from multiple institutions treated with gamma knife RS showed an overall hearing preservation rate of about $51 \%$ 3-4 years after treatment. ${ }^{(22)}$ In our study, it is interesting to note that the rate of hearing preservation was much lower (50\%) in the hypofractionated treatment group. Similar to our findings, a report of 29 patients treated with $7 \times 4$ Gy reported a five-year actuarial Class A/B (pure-tone thresholds $\leq 50 \mathrm{~dB}$ and speech discrimination $\leq 50 \%$ ) hearing preservation rate of $50 \%$. ${ }^{(12)}$ Another report of 27 patients who underwent hypo-FSRT found that none of the patients treated with 20-24 Gy in 5-6 fractions preserved serviceable hearing. ${ }^{(23)}$ However, another report of patients treated with cyberknife in 2-5 fractions (14-19.5 Gy) showed that seven out of eight patients with baseline serviceable hearing retained their hearing, ${ }^{(16)}$ although the doses used were lower compared with other studies. Hypofractionated RS needs to be further evaluated for hearing preservation safety.

Our rates of cranial neuropathies were low, with more than $90 \%$ of patients having no worsening or new facial and trigeminal nerve palsies, or worsening disequilibrium. This is comparable to other studies, as outlined in Murphy and Suh's review of FSRT and RS, which reported a cranial nerve $\mathrm{V}$ and $\mathrm{VII}$ preservation rate of more than $95 \%$. $^{(24)}$
It has been reported that the general risk of hearing deterioration after surgery is $30 \%-50 \%$ and that of local control is $80 \%-98 \%$, and the risk of mortality has been cited as less than $2 \%$ in most series. ${ }^{(25,26)}$ For patients who are not surgical or general anaesthetic candidates, RT is a good option. Even for fit patients, some opine that RS is the treatment of choice for smaller uncomplicated tumours. A systematic review of only controlled studies that compared microsurgery, RS and fractionated RT concluded that for solitary VS up to $30 \mathrm{~mm}$ in cisternal diameter, RS showed better outcomes compared to microsurgery, as there were no direct mortality and surgical or anaesthetic complications, and patients had better preservation of facial nerve and hearing, as well as better quality of life. ${ }^{(3)}$ However, the review could not find any randomised or controlled studies on fractionated RT. Although the systematic review included six studies comparing RS and microsurgery, only four studies $^{(27-30)}$ were deemed to be of good quality with no relevant bias identified. In these four studies, facial nerve was intact in $67 \%-82 \%$ and $91 \%-100 \%$ of microsurgical and RS patients, respectively, while hearing preservation was $0 \%-36 \%$ and $32 \%-$ $75 \%$, respectively. Hence, we suggest that small, uncomplicated tumours be treated with RS. However, for patients who have tumours with hydrocephalus, surgery is the preferred option, as they will benefit from the immediate relief of pressure.

In choosing between FSRT and RS, there are a few factors to take into consideration. Some clinicians believe that due to the radiobiological benefit of a smaller dose per fraction, FSRT has the benefits of hearing preservation and increased safety for larger tumours. As reported in a series of 99 patients treated at Addenbrookes Hospital, United Kingdom, with 50 Gy in 30 fractions conformal RT, the actuarial local control rate was $96.9 \%$, hearing preservation $100 \%$, facial nerve preservation $96.8 \%$ and trigeminal nerve preservation $100 \% .{ }^{(31)}$ In a review published in 2014, Jian et al concluded that fractionated RT is an excellent treatment alternative to RS, especially in patients with larger tumours, largely due to its excellent tumour control and toxicity profile. ${ }^{(32)}$ On the other hand, FSRT is logistically more inconvenient, as it takes up more of the patient's time and may also add strain to busy treatment units. Unfortunately, there is no direct study comparing FSRT and RS in the literature. Most studies are retrospective, and subjects in the RS arm had smaller and less complicated tumours. In a study of 104 patients treated with RS, conventional FSRT (45-50.4 Gy in 35-28 fractions) or hypo-FSRT (20 Gy in 5 fractions), the five-year progression-free and cranial nerve toxicity rates were equivalent. However, there was no comparison of tumour size, and the patients treated with FSRT had better baseline hearing as well as facial and trigeminal nerve functions. ${ }^{(33)}$ Studies looking at outcomes and toxicities of FSRT reported good local control, good hearing and cranial nerve preservation, with hearing preservation of $73 \%-98 \%,(7,9,31,34-37)$ as well as better hearing preservation at the one-year follow-up compared to RS. ${ }^{(7)}$ With a longer follow-up of 67 months, a pooled German study found no difference in loss of useful hearing rates between the FSRT and stereotactic RS groups (14\% vs. $16 \%)^{(5)}$ However, it is admittedly difficult to compare the rate of hearing 
preservation among different studies due to the different recording methods used (such as the Gardner-Robertson scale, word discrimination method and subjective reporting). Although RS is not recommended for large VSs, there was a single case report of a large VS $(3.8 \mathrm{~cm}$ in diameter) treated with FSRT following VP shunt placement that showed good tumour control and no cranial nerve or hearing morbidity at 63 months of follow-up; the authors thus concluded that FSRT was a potential noninvasive treatment modality for larger tumours. ${ }^{(38)}$

To combine the efficacy of single-fraction RS and the supposed safety of FSRT, some clinicians have proposed treatment with hypofractionated RS. In the present study, patients who received hypofractionated RS had larger tumours and tended to be in Koos Grade 3 or 4, as it is believed that hypofractionated RS may confer more clinical safety for riskier tumours. A Japanese series of 25 patients with 26 VSs treated with hypofractionated RS showed a seven-year progression-free rate of $95 \%$. Of the 12 patients who had PTA $<50 \mathrm{~dB}$ before RS, the mean PTA before and after hypofractionated RS was $29.8 \mathrm{~dB}$ and $57.1 \mathrm{~dB}$, respectively. 2 (7.7\%) patients developed Grade 2 facial nerve disorder. ${ }^{(14)}$ In a German series of 29 subjects treated with $7 \times$ $4 \mathrm{~Gy}$, the five-year hearing preservation rate was $50 \%$. It also reported a transient increase in tumour volume in 17 out of 29 patients; however, it was unclear how tumour volume increase was defined. After a median follow-up period of 89.5 months, only one patient showed radiological progression and no patient required intervention. ${ }^{(12)}$ Another study on benign skull base tumours, which included ten VSs treated with $5 \times 5$ Gy, local control and cranial nerve function preservation were reported in all the patients. ${ }^{(13)}$ Other series on hypofractionated RS have reported similar outcomes, including a Japanese study ${ }^{(23)}$ and Taiwanese ${ }^{(11)}$ study, both of which reported no permanent facial or trigeminal nerve morbidity and no salvage surgery. Another study reported only one case each of vertigo, tongue paraesthesia and trigeminal neuralgia among the 33 cases of large VSs. ${ }^{(16)}$ With these studies in mind, the safety profile (excluding hearing preservation) of hypofractionated RS appears to be quite established, although experience with hypofractionated RS has been reported much less frequently than single-fraction RS.

Malignant transformation of a tumour or secondary neoplasm from irradiation exists, although the risk is small. Balasubramaniam et $\mathrm{al}^{\prime} \mathrm{s}^{(39)}$ review of secondary tumours after stereotactic RT found that only 20 cases have been reported as of 2007. Of the 20 patients, 14 had VS. Eight of the VS patients had NF-2, six of whom had pathological confirmation of malignant transformation 2-6 years after RT when they presented with accelerated tumour growth. The histology was malignant nerve sheath tumour in most of the cases. ${ }^{(39)}$ In another report of 440 patients who were followed up for more than ten years after gamma knife surgery for VS, only one developed malignant transformation. ${ }^{(10)}$ Therefore, although the risk of secondary tumours is small, this information must be conveyed to patients, especially younger or NF-2 patients, prior to RT.

Another rare but serious complication of RT is brainstem radiation necrosis. A study of 93 VS patients treated with FSRT reported a single case of probable radionecrosis of the brainstem with serious complications that manifested nine months after FSRT. This patient was found to have been treated within brainstem tolerance and thus the authors postulated that the patient's previous surgical excision of the tumour could have reduced his brainstem tolerance. ${ }^{(36)}$

Bearing the pros and cons of the various treatment options in mind, observation may sometimes be a valid and the most reasonable option for some patients, given the indolent natural history of VS, especially in patients with a limited estimated lifespan or poorer performance status, or those who are asymptomatic or incidentally diagnosed during brain MR imaging. In the current series, 19 patients had RT upon diagnosis of VS. Although all 19 were symptomatic, their tumours may not have progressed during the follow-up period.

Going forward, prospective trials comparing the different treatment modalities are required, although with high tumour control and low toxicity rates across all treatment options, obtaining statistically significant results may be difficult, as a large number of patients will be needed. To better compare between studies, standardising the reporting of tumour size $\left(\mathrm{cm}^{3}\right.$ or maximal diameter) and tumour control, such as RECIST criteria and toxicity reporting, should be undertaken for future trials or cohort reporting.

The majority of the VS patients in the current study were treated only after a period of initial observation, having shown evidence of size progression. Hughes et $\mathrm{al}^{(40)}$ reported that in a retrospective series of 59 patients who were chosen for initial observation, only 11 (19\%) eventually required intervention. They also found that tumours that had already extended into the cerebellopontine angle had a significantly faster rate of growth than intracanalicular tumours. ${ }^{(40)}$

The present study was not without limitations. Firstly, our hearing preservation results were subject to bias, as only 11 of our patients had serviceable hearing before treatment (our institution's practice is to offer FSRT only to patients with good baseline hearing). Secondly, toxicity scoring, facial nerve palsy, post-RS disequilibrium and trigeminal neuropathy were based on subjective reporting of symptoms and not objectively measured with clinical tests or scores such as electronystagmography or House-Brackmann score. As grading of facial nerve function was not rigorously done, the low rate of facial nerve palsy after RS may not be accurate. Finally, the decision to treat with singlefraction or hypofractionated RS was not made based on definite or clear-cut departmental guidelines, except that larger tumours with more brainstem mass effect were more likely to be treated with a hypofractionated regimen.

In conclusion, small, uncomplicated but enlarging VSs should be treated with RS, although observation is an option for a selected subgroup, especially older patients or those with small, asymptomatic and slow-growing tumours. Observation can also be practised after subtotal resection, as shown in a report in which only 18 out of 116 patients had recurrence after subtotal resection. ${ }^{(41)} \mathrm{RS}$ is efficacious with good safety records. Microsurgery can be reserved for larger, more complicated VSs, 
with adjuvant RS an option for recurrences. Between FSRT and $\mathrm{RS}$, it is unclear if the former offers better hearing preservation in the long run.

\section{ACKNOWLEDGEMENTS}

We would like to thank Dr Chua Eu Tiong and his team of radiation oncologists from the Department of Radiation Oncology, National Cancer Centre Singapore, and SingHealth neurosurgeons and physicists who were involved in the care of the patients in this study.

\section{REFERENCES}

1. Muzevic D, Legcevic J, Splavski B, Cayé-Thomasen P. Stereotactic radiotherapy for vestibular schwannoma. Cochrane Database Syst Rev 2014; (12):CD009897.

2. Breivik CN, Nilsen RM, Myrseth E, et al. Conservative management or gamma knife radiosurgery for vestibular schwannoma: tumor growth, symptoms, and quality of life. Neurosurgery 2013; 73:48-56; discussion 56-7.

3. Wolbers JG, Dallenga AH, Mendez Romero A, van Linge A. What intervention is best practice for vestibular schwannomas? A systematic review of controlled studies. BMJ Open 2013; 3(2), pii: e001345.

4. Neurosurgeon Coding for Stereotactic procedures. Available at: https://www. varian.com/sites/default/files/resource_attachments/NeurosurgeryCodingGuide RAD10297C January2016.pdf. Accessed August 28, 2018.

5. Combs SE, Engelhard C, Kopp C, et al. Long-term outcome after highly advanced single-dose or fractionated radiotherapy in patients with vestibula schwannomas - pooled results from 3 large German centers. Radiother Oncol 2015; 114:378-83.

6. Eisenhauer EA, Therasse P, Bogaerts J, et al. New response evaluation criteria in solid tumours: revised RECIST guideline (version 1.1). Eur J Cancer 2009; 45:228-47.

7. Andrews DW, Suarez O, Goldman HW, et al. Stereotactic radiosurgery and fractionated stereotactic radiotherapy for the treatment of acoustic schwannomas: comparative observations of 125 patients treated at one institution. Int J Radiat Oncol Biol Phys 2001; 50:1265-78.

8. Benghiat $\mathrm{H}$, Heyes $\mathrm{G}$, Nightingale $\mathrm{P}$, et al. Linear accelerator stereotactic radiosurgery for vestibular schwannomas: a UK series. Clin Oncol (R Coll Radiol) 2014 26:309-15.

9. Chan AW, Black P, Ojemann RG, et al. Stereotactic radiotherapy for vestibular schwannomas: favorable outcome with minimal toxicity. Neurosurgery 2005; 57:60-70

10. Hasegawa T, Kida Y, Kato T, et al. Long-term safety and efficacy of stereotactic radiosurgery for vestibular schwannomas: evaluation of 440 patients more than 10 years after treatment with Gamma Knife surgery. J Neurosurg 2013; 118:557-65.

11. Ju DT, Lin JW, Lin MS, et al. Hypofractionated CyberKnife stereotactic radiosurgery for acoustic neuromas with and without association to neurofibromatosis Type 2. Acta Neurochir Suppl 2008; 101:169-73.

12. Kranzinger M, Zehentmayr F, Fastner G, et al. Hypofractionated stereotactic radiotherapy of acoustic neuroma: volume changes and hearing results after 89-month median follow-up. Strahlenther Onkol 2014; 190:798-805.

13. Mahadevan A, Floyd S, Wong E, Chan C, Kasper E. Clinical outcome after hypofractionated stereotactic radiotherapy (HSRT) for benign skull base tumors Comput Aided Surg 2011; 16:112-20.

14. Morimoto M, Yoshioka Y, Kotsuma T, et al. Hypofractionated stereotactic radiation therapy in three to five fractions for vestibular schwannoma. Jpn J Clin Oncol 2013; 43:805-12.

15. Iwai Y, Ishibashi K, Watanabe Y, Uemura G, Yamanaka K. Functional preservation after planned partial resection followed by Gamma Knife radiosurgery for large vestibular schwannomas. World Neurosurg 2015; 84:292-300.

16. Casentini L, Fornezza U, Perini Z, Perissinotto E, Colombo F. Multisession stereotactic radiosurgery for large vestibular schwannomas. J Neurosurg 2015; 122:818-24.
17. Aoyama H, Onodera S, Takeichi N, et al. Symptomatic outcomes in relation to tumor expansion after fractionated stereotactic radiation therapy for vestibular schwannomas: single-institutional long-term experience. Int J Radiat Oncol Biol Phys 2013; 85:329-34.

18. Norén G, Greitz D, Hirsch A, Lax I. Gamma knife surgery in acoustic tumours. Acta Neurochir Suppl (Wien) 1993; 58:104-7.

19. Kapoor S, Batra S, Carson K, et al. Long-term outcomes of vestibular schwannomas treated with fractionated stereotactic radiotherapy: an institutional experience. Int J Radiat Oncol Biol Phys 2011; 81:647-53.

20. Koos W, Kletter G, Bock F, Salah S. [Microsurgery of bilateral tumors of the cerebellopontile angle]. Neurochirurgie 1972; 18:75-6. French.

21. Yang $\mathrm{HC}$, Kano $\mathrm{H}$, Awan NR, et al. Gamma Knife radiosurgery for larger-volume vestibular schwannomas: clinical article. J Neurosurg 2013; 119 Suppl:801-7.

22. Yang I, Sughrue ME, Han SJ, et al. A comprehensive analysis of hearing preservation after radiosurgery for vestibular schwannoma: clinical article. J Neurosurg 2013; 119 Suppl:851-9.

23. Sakanaka K, Mizowaki T, Arakawa Y, et al. Hypofractionated stereotactic radiotherapy for acoustic neuromas: safety and effectiveness over 8 years of experience. Int J Clin Oncol 2011; 16:27-32.

24. Murphy ES, Suh JH. Radiotherapy for vestibular schwannomas: a critical review. Int J Radiat Oncol Biol Phys 2011; 79:985-97.

25. Arthurs BJ, Fairbanks RK, Demakas JJ, et al. A review of treatment modalities for vestibular schwannoma. Neurosurg Rev 2011; 34:265-79.

26. Kaylie DM, McMenomey SO. Microsurgery vs gamma knife radiosurgery for the treatment of vestibular schwannomas. Arch Otolaryngol Head Neck Surg 2003; 129:903-6.

27. Myrseth E, Møller P, Pedersen PH, Lund-Johansen M. Vestibular schwannoma: surgery or gamma knife radiosurgery? A prospective, nonrandomized study. Neurosurgery 2009; 64:654-63.

28. Pollock BE, Lunsford LD, Kondziolka D, et al. Outcome analysis of acoustic neuroma management: a comparison of microsurgery and stereotactic radiosurgery. Neurosurgery 1995; 36:215-29.

29. Régis J, Pellet W, Delsanti C, et al. Functional outcome after gamma knife surgery or microsurgery for vestibular schwannomas. J Neurosurg 2013; 119 Suppl:1091-100.

30. Myrseth E, Møller P, Pedersen PH, et al. Vestibular schwannomas: clinical results and quality of life after microsurgery or gamma knife radiosurgery. Neurosurgery 2005; 56:927-35.

31. Horan G, Whitfield GA, Burton KE, Burnet NG, Jefferies SJ. Fractionated conformal radiotherapy in vestibular schwannoma: early results from a single centre. Clin Oncol (R Coll Radiol) 2007; 19:517-22.

32. Jian BJ, Kaur G, Sayegh ET, et al. Fractionated radiation therapy for vestibular schwannoma. J Clin Neurosci 2014; 21:1083-8.

33. Anderson BM, Khuntia D, Bentzen SM, et al. Single institution experience treating 104 vestibular schwannomas with fractionated stereotactic radiation therapy or stereotactic radiosurgery. J Neurooncol 2014; 116:187-93.

34. Combs SE, Volk S, Schulz-Ertner D, et al. Management of acoustic neuromas with fractionated stereotactic radiotherapy (FSRT): long-term results in 106 patients treated in a single institution. Int J Radiat Oncol Biol Phys 2005; 63:75-81.

35. Fuss $M$, Debus J, Lohr $F$, et al. Conventionally fractionated stereotactic radiotherapy (FSRT) for acoustic neuromas. Int J Radiat Oncol Biol Phys 2000; 48:1381-7.

36. Woolf DK, Williams M, Goh CL, et al. Fractionated stereotactic radiotherapy for acoustic neuromas: long-term outcomes. Clin Oncol (R Coll Radiol) 2013; 25:734-8.

37. Selch MT, Pedroso A, Lee SP, et al. Stereotactic radiotherapy for the treatment of acoustic neuromas. J Neurosurg 2004; 101 Suppl 3:362-72.

38. McClelland S 3rd, Gerbi BJ, Cho KH, Hall WA. The treatment of a large acoustic tumor with fractionated stereotactic radiotherapy. J Robot Surg 2007; 1:227-30.

39. Balasubramaniam A, Shannon P, Hodaie M, et al. Glioblastoma multiforme after stereotactic radiotherapy for acoustic neuroma: case report and review of the literature. Neuro Oncol 2007; 9:447-53.

40. Hughes M, Skilbeck C, Saeed S, Bradford R. Expectant management of vestibular schwannoma: a retrospective multivariate analysis of tumor growth and outcome. Skull Base 2011; 21:295-302.

41. Cerullo L, Grutsch J, Osterdock R. Recurrence of vestibular (acoustic) schwannomas in surgical patients where preservation of facial and cochlear nerve is the priority. Br J Neurosurg 1998; 12:547-52. 